\title{
Revisión de la validez del coeficiente de seguridad, propuesto por EHE-08, para la estimación de la vida útil de cálculo en estructuras de edificación sometidas a la acción de cloruros
}

\author{
Revision of validity for the factor of safety (EHE-o8) of the service life \\ in structures under chlorides
}

\author{
E. R. Mosquera-Rey ${ }^{(*)}$
}

RESUMEN

El objetivo de este artículo es la necesidad de revisar el coeficiente de seguridad de la vida útil propuesto en el anejo $9^{\circ}$ de EHE-08. En concreto, para la corrosión por cloruros bajo ciertas condiciones, se observa que los valores son claramente escasos o inseguros. En la metodología empleada solo se pondera la vida útil de proyecto. El resto de las variables (concentraciones, coeficiente de difusión, etc.), tienen carácter determinista y no se les atribuye aleatoriedad o la tienen en cuenta de forma implícita; además sus valores pueden variar, lo que se conoce como falta de invarianza de las medidas de seguridad. En estas condiciones la seguridad puede no ser única cuando la determinación del nivel de seguridad debe ser un invariante.

Se propone un coeficiente de seguridad en base a un determinado índice de fiabilidad ( $\beta$ ) y un coeficiente de variación (CV), para una supuesta variable aleatoria $(\mathrm{t})$.

Palabras clave: Durabilidad; fiabilidad; metodología probabilista; vida útil.

\section{ABSTRACT}

This paper presents a revision of the factor of safety of the service life suggested in the annex 9 of the Spanish Code on the Structural Concrete (EHE-O8). The values in the results achieved in the particular case of corrosion by chlorides under certain conditions are clearly unsafe and not enough. The methodology used is similar to that of the security global method since only the service life of the project is considered. Any other variable (critical concentration, surface concentration, diffusion coefficient) is deterministic. The randomness can be implicit or not and the representatives values can vary which known as lack of invariance in the security measures. From the point of view of structural reliability, the level of security must be an invariant.

This paper proposes a factor of safety based on a certain reliability index ( $\beta$ ) and a coefficient of variation (CV) for a supposed random variable.

Keywords: Durability; reliability; probabilistic methodology; lifespan.

(*) Universidad de A Coruña (España).

Persona de contacto/Corresponding author: emilio.mosquera@udc.es (E. R. Mosquera-Rey)

Cómo citar este artículo/Citation: Mosquera-Rey, E. R. (2015). Revisión de la validez del coeficiente de seguridad, propuesto por EHE-08, para la estimación de la vida útil de cálculo en estructuras de edificación sometidas a la acción de cloruros. Informes de la Construcción, 67(538): eo82, doi: http://dx.doi.org/10.3989/ic.13.27.

Licencia / License: Salvo indicación contraria, todos los contenidos de la edición electrónica de Informes de la Construcción se distribuyen bajo una licencia de uso y distribución Creative Commons Reconocimiento no Comercial 3.o. España (cc-by-nc). 


\section{INTRODUCCIÓN Y PLANTEAMIENTOS INICIALES}

\subsection{Algo sobre la vida útil}

En general, cualquier diseño de un proceso, requiere o exige la definición clara del final de la vida de servicio. Sin embargo, de las definiciones de las distintas normas (1) (2) (3) se determina el punto final en el tiempo pero no se llega a valores concretos y universales claros de las condiciones de deterioro de las propiedades del material. Los deterioros se suelen dividir en clases, por ejemplo: según su apariencia, según su función, según su seguridad, etc. (4) (5). En estructuras ejecutadas, la demanda de cumplimiento o requerimiento se conoce como vida residual (6) (7).

Desde el punto de vista de deterioro de una estructura de hormigón armado por efecto de la corrosión por cloruros se puede considerar:

a) Vida útil de proyecto, se corresponde con el tiempo que va desde el inicio de la construcción hasta la despasivación de las armaduras, dada en general por la llegada de los cloruros a las mismas con cierta concentración.

b) Vida útil de servicio o utilización, se corresponde con el tiempo que va desde el inicio de la construcción hasta que alcanza un determinado estado de fisuración del hormigón, aparecen manchas superficiales u ocurren desprendimientos del revestimiento.

c) Vida útil residual, se corresponde al período de tiempo entre la fecha de una cierta inspección y la fecha límite de vida útil previamente establecida.

En estas condiciones, el término de vida útil de proyecto se podría sustituir por el de durabilidad, en alusión a una cantidad de años medibles y cuantificables (1).

\subsection{Verificación de la vida útil}

El límite inaceptable de deterioro depende del tipo de elemento estructural y de las repercusiones sociales y económicas. Bajo ciertas condiciones se podría considerar como límite, el tiempo de despasivación del acero; y en otras condiciones: el inicio de la fisuración, el estallido, la pérdida de sección del acero, la pérdida de adherencia o la pérdida de capacidad portante, etc. (etapas) (7) (8). Paulo Helene, describe los principales métodos de análisis en base: a experiencias anteriores o en base a ensayos acelerados. Desde el concepto de las variables, los modelos pueden ser deterministas o probabilistas, básicamente, en función de la aleatoriedad de las variables. En los últimos años se han desarrollado múltiples modelos de penetración de cloruros y diseño de vida útil. Por destacar algunos significativos: C. Andrade (1994) enfoque esquemático en una etapa, Tang y Nilson (1994) modelo numérico en elementos finitos, Yamamoto (1995), Sarja y Versikari (1996) modelos deterministas y probabilistas, Metha (1997), Castañeda (1997), Najesh y Bishwajit (1998) difusión en hormigones saturados y no saturados, Liu y Weyers (1998) modelo empírico no lineal, Hansen y Saouma(1999) aplicación Abaqus, Hooton (2002) considerando el efecto del curado, Siemes y de Vries (2002) probabilísticos en cinco etapas, Rostam (2003) y A. Lindvall (2005) probabilísticos, Zhang (2006) considerando la etapa de iniciación, Castro-Borges y Helene (2007) esquemático hasta con siete etapas, Baroghel-Bouny, Thai Luang Nguyen
(2008) modelo multinivel etc. (8). Un aspecto importante de los métodos basados en la difusión lo constituye la consideración de que ciertos valores sean variables o constantes en el tiempo por ejemplo Mangat, Uji, Mejlbro etc. Izquierdo (2003) en base probabilista sobre coeficientes parciales. Incluso se han propuesto modelos especulativos basados en técnicas Focus Group como el de J. Oliveira (2002). Contribuciones especiales por la trascendencia normativa son: Bentz (2008) Life-365, Siemes COWI (1999) DuraCrete (2000), Peter Schiessl y otros (2006) bulletin 34 fib Model Code etc. (9).

Con carácter general el modelo más difundido de predicción de vida útil es debido a Tuutti (1982), (3) (10) (11) (12).Ver Figura 1.

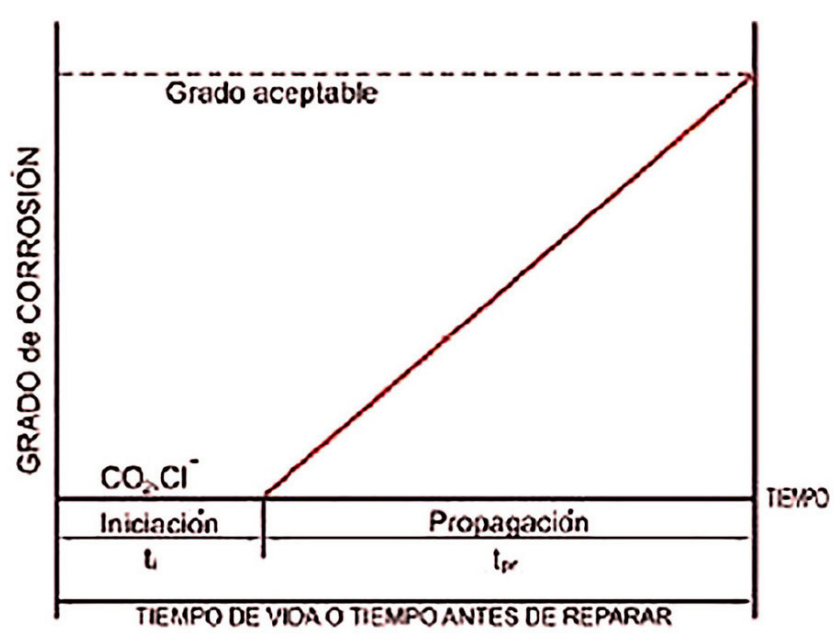

Figura 1. Representación esquemática del proceso de corrosión del acero en hormigón (Modelo deTuutti).

Este modelo define dos períodos claramente diferenciados, el de iniciación y el de propagación. El primero está caracterizado por el tiempo de penetración de las sustancias agresivas medioambientales ( $\mathrm{CO}_{2}, \mathrm{Cl}^{-}$, etc.) hasta alcanzar la despasivación de las armaduras. Este período de iniciación no presenta la corrosión en estado activo y su límite es claro en el caso de cloruros (una cierta concentración crítica en las armaduras).

El período de propagación es el tiempo desde la despasivación hasta un cierto grado de deterioro. Este segundo período es difícil de modelar y la velocidad de corrosión va a depender fundamentalmente de la disponibilidad de agua y oxígeno en la interfase acero/hormigón (8). El límite de deterioro estará asociado a un determinado tipo de estado límite (servicio/último), dependiendo del nivel de deterioro admitido, que estará asociado a su vez a un determinado índice de fiabilidad (o probabilidad de fallo). En caso de armaduras activas, este periodo no ha de ser considerado, $\mathrm{t}_{\mathrm{p}}=\mathrm{o}(7)(13)$.

En estas condiciones la verificación de la vida útil para las estructuras de hormigón armado afectadas por cloruros en los ambientes IIIa hasta $500 \mathrm{~m}$ y IIIc, se caracterizan por tener una velocidad de corrosión alta, en términos generales entre 20 y $50 \mu \mathrm{m} /$ año. Esto supone un periodo de propagación corto o poco significativo respecto de la vida útil. 
Por todo lo anteriormente expuesto la mayoría de las predicciones de la vida útil de las estructuras se basan en estimar el período de iniciación a través de una metodología probabilista e índices de fiabilidad que podrían estar entre $\beta=1 \mathrm{y}$ $\beta=1,5$ (7) (13). Estimándose como más razonable $\beta=1,3$ que proporciona una probabilidad de fallo $P f=10 \%$

\subsection{Aleatoriedad de la vida útil}

Los fenómenos naturales son claramente aleatorios y su consideración como constantes en el tiempo provoca desviaciones importantes en los resultados. Por esta razón existe un amplio consenso internacional en la consideración del estado límite de despasivación como vida útil a través de un formato probabilista vinculado a niveles de fiabilidad en órdenes de fallo anteriormente expuestas (13).

La consideración probabilista supone la valoración de las incertidumbres que todo este proceso conlleva, (2) (14) por lo que cualquier estimación en términos semiprobabilistas con valores medios de las variables básicas, presupone probabilidades de fallo claramente indeseables.

El anejo 9 de EHE-o8 considera el estado límite de durabilidad como un procedimiento de carácter semiprobabilista en donde estima la vida útil y la compara con la vida útil de proyecto. Este carácter obliga a que las variables utilizadas para la estimación sean características o nominales. Sin embargo al menos en 3 de las variables se puede cuestionar su carácter de valor característico.

a) La tabla A.9.4. de Coeficientes $\mathrm{D}$ (to) proporciona valores del coeficiente de difusión a 28 días que tienen un alto grado de coincidencia con los valores iniciales propuestos en el documento (13) Model Code for Service Life Design. fib-Boletín 34. Estos son valores medios que representan una distribución aleatoria normal con una desviación típica cuyo coeficiente de variación es o.2. Son valores obtenidos mediante un ensayo acelerado de migración.

b) El factor de edad «n» tiene un carácter determinante en el coeficiente de difusión de cloruros. El anejo le atribuye un valor igual a 0,5 (a falta de valores específicos obtenidos mediante ensayos). El Model Code for Service Life Design. fib-Boletín 34 (13) establece como valores probabilistas los de la Tabla 1.

Tabla 1. Valores probabilistas "Model Code" para: Distribución beta, $\mathrm{m}$ valor medio, $\mathrm{s}$ desviación estándar (13)

\begin{tabular}{|l|l|}
\hline \multicolumn{1}{|c|}{ Hormigón } & \multicolumn{1}{c|}{$\begin{array}{c}\text { "n" exponente } \\
\text { de edad }\end{array}$} \\
\hline $\begin{array}{l}\text { Cemento Portland } \\
\text { CEM I; } 0,40<\mathrm{a} / \mathrm{c}<0,60\end{array}$ & Beta $\mathrm{m}=0,3, \mathrm{~s}=0,12$ \\
\hline $\begin{array}{l}\text { Cemento Portland con cenizas volantes } \\
\mathrm{K}=0,5: \mathrm{I} ; \mathrm{o}, 4 \mathrm{O}<\mathrm{a} / \mathrm{c}<0,62\end{array}$ & Beta $\mathrm{m}=0,6, \mathrm{~s}=0,15$ \\
\hline $\begin{array}{l}\text { Cemento con } \mathrm{Humo} \text { de sílice } \\
\text { CEM III } / \mathrm{B} ; \mathrm{o}, 40<\mathrm{a} / \mathrm{c}<0,60\end{array}$ & Beta $\mathrm{m}=0,45, \mathrm{~s}=0,20$ \\
\hline
\end{tabular}

Como conclusión, a la vista de la Tabla 1, el valor de 0,5 puede ser considerado como un valor medio independiente del tipo de cemento y de sus adiciones. c) La concentración crítica de cloruros se establece en un valor del o,6 \% en peso de cemento, caso de corrosión en armaduras pasivas. El Model Code for Service Life Design. fib-Boletín 34 establece, para su valoración probabilista una distribución de probabilidades beta con los siguientes valores:

- media = 0,60 \% en peso de cemento

- desviación estándar = 0,15\% en peso de cemento

- mínimo valor $=0,2 \%$ en peso de cemento

- máximo valor $=2,0 \%$ en peso de cemento

Pues bien, cualquier cálculo efectuado con estos parámetros proporcionará una estimación media de la vida útil. Por tanto, toda la incertidumbre del proceso está confiada al coeficiente de seguridad propuesto por la normativa de valor 1,1 .

\subsection{Contenido inicial de cloruros, $C_{b}$}

El contenido inicial de cloruros, aportados por la masa de hormigón, es un parámetro de carácter constante y uniforme, lo que le da una incidencia variable en el cálculo de los recubrimientos (15). En principio es un parámetro sobre el que es posible actuar desde el diseño del material. Es lógico pensar que si se quiere construir una estructura en un ambiente de exposición de cloruros, el diseño de la masa de hormigón debería contemplar una dosificación con un contenido mínimo de cloruros, por lo que este parámetro estaría controlado en el proceso de diseño del material.

Efectuados cálculos (9) para la situación pésima, la de ambiente con mayor concentración superficial de cloruros, y para contenido máximo de cloruros de $0,4 \%$ en peso de cemento, la variación del recubrimiento es del orden del 25 al 30 \% más que si el contenido de cloruros inicial fuese o.

La conclusión se hace evidente y acorde con literatura especializada (7), que en el caso de situación de agresión importante, la concentración inicial se debe limitar por diseño del material y puede ser despreciada para el cálculo del tiempo de despasivación de la armadura.

En la realidad, a través de la experiencia profesional (9), se observa que no se comprueba, de forma sistemática, la concentración inicial de cloruros en la masa fresca del hormigón, ni por las centrales de fabricación de hormigón, ni por los laboratorios de control, por entender que es un parámetro con poca relevancia en el proceso. Sin embargo, es cuando menos sospechoso, el alto grado de corrosión no explicable por los procesos de difusión y un posible incumplimiento de la normativa.

\section{ANÁLISIS SOBRE VIDA ÚTIL}

\subsection{Verificaciones Deterministas}

A continuación se resumen los cálculos deterministas realizados (9).

Al objeto de comprobar la realidad anteriormente planteada y observar el comportamiento (con la metodología propuesta por el anejo $9^{\circ}$ ), respecto del recubrimiento de los distintos hormigones en los ambientes IIIa-500 y IIIc, con conteni- 
dos iniciales de cloruros, con valores extremos: $\mathrm{C}_{\mathrm{b}}=0,4 \% \mathrm{y}$ $\mathrm{C}_{\mathrm{b}}=\mathrm{o} \% \mathrm{y}$ bajo las siguientes condiciones:

- Relaciones agua/cemento: 0,40-0,45-0,50 y 0,55

- Contenido de cemento: 300-325-350-375 y $400 \mathrm{~kg} / \mathrm{m}^{3}$

- Concentración Crítica de despasivación: o,6 \% en peso de cemento

- Referencia temporal: 50 años

- Valores de coef. de difusión y concentración superficial los correspondientes del anejo $9^{\circ}$

Para el desarrollo de los cálculos se ha utilizado la aplicación informática Mathcad-14, (16) aplicación diseñada para la resolución productiva de problemas de ingeniería y presentación de soluciones. Permite combinar texto, matemáticas y gráficos en un único entorno de hoja de trabajo, visualiza, ilustra y verifica. Los resultados se muestran en las Figuras 2 y 3 las que se ilustran a continuación.

Dónde:

CC: Vector de contenido de cemento en $\mathrm{Kg} / \mathrm{m}^{3}$

$A / C$ : Vector de relación agua-cemento.

$d$ : Matriz de recubrimientos mínimos requeridos bajo las condiciones estudiadas en $\mathrm{mm}$.

Ejemplo en Figura 2: Ambiente IIIa-50o., Contenido inicial de cloruros: $C_{\mathrm{b}}=$ o, Cemento CEM I

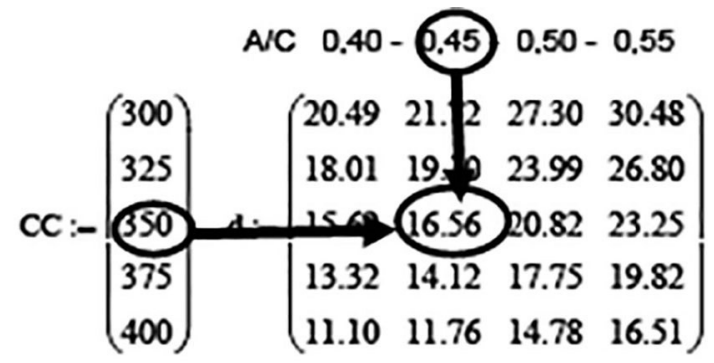

Figura 2. Ejemplo de matrices.

Representación de la matriz recubrimientos mínimos en Figura 3 .

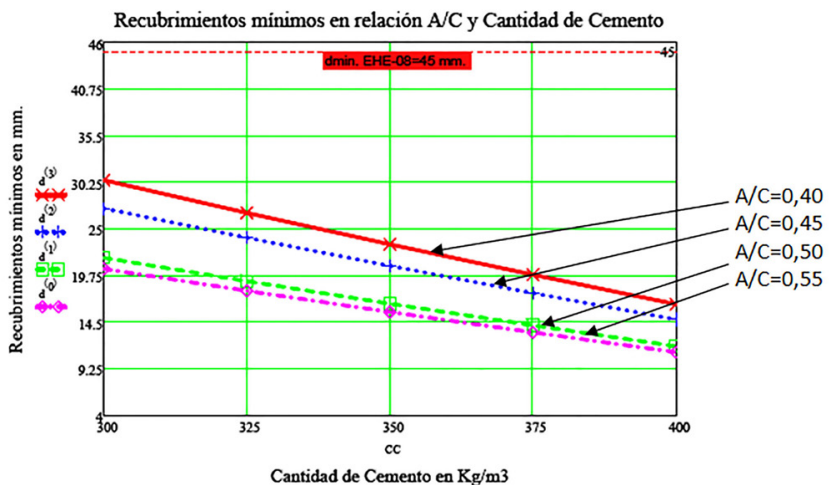

Figura 3. Representación de matriz de recubrimientos mínimos.

Las Figura 4 y 5 muestran los resultados numérico-gráficos de los cálculos desarrollados.
Del mismo modo se realiza un estudio semiprobabilista para $t=50$ años con el coeficiente de seguridad propuesto en el anejo $9^{\circ}, \gamma=1,1$.

Como resumen de este análisis se presenta la siguiente Tabla 2, donde se observa la gran incertidumbre a la que pueden conducir tales cálculos.

Tabla 2. Análisis semiprobabilista - Vida útil 50 años, Tipo de Ambiente IIIa-500 - Condiciones Extremas

\begin{tabular}{|l|l|c|c|c|}
\hline \multirow{2}{*}{$\begin{array}{c}\text { Tipo de } \\
\text { Cemento }\end{array}$} & $\begin{array}{c}\text { Condiciones } \\
\text { de Dosifica- } \\
\text { ción }\end{array}$ & $\begin{array}{c}\text { Recubri- } \\
\text { miento míni- } \\
\text { mo estimado } \\
\text { (mm) }\end{array}$ & $\begin{array}{c}\text { Recubri- } \\
\text { miento míni- } \\
\text { mos/ art.37 } \\
\text { (mm.) }\end{array}$ & $\begin{array}{c}\text { Tiempo } \\
\text { necesario } \\
\text { hasta la des- } \\
\text { pasivación } \\
\text { (años) }\end{array}$ \\
\hline \multirow{2}{*}{ CEM I } & Optimas & 11 & 45 & 13500 \\
\cline { 2 - 5 } & Pésimas & 31 & 45 & 240 \\
\hline CEM & Optimas & 9 & 45 & 32000 \\
\cline { 2 - 5 } IIA-V & Pésimas & 23 & 45 & 780 \\
\hline \multirow{2}{*}{ CEM III } & Optimas & 4,5 & 25 & 51000 \\
\cline { 2 - 5 } & Pésimas & 12 & 25 & 980 \\
\hline
\end{tabular}

Dosificación Óptima. Relación a $/ \mathrm{c}=0,40$ y cantidad de cemento $=400 \mathrm{~kg} / \mathrm{m}^{3}$ Dosificación Pésima. Relación a $/ \mathrm{c}=0,55$ y cantidad de cemento $=300 \mathrm{~kg} / \mathrm{m}^{3}$

A modo de resumen comparativo se presentan en la Tabla 3 los rangos de los recubrimientos según el ambiente y tipo de cemento.

Tabla 3. Resumen comparativo por años

\begin{tabular}{|c|c|c|}
\hline \multicolumn{3}{|c|}{50 años } \\
\hline & Ambiente IIIa- 500m & Ambiente IIIc \\
\hline CEM I & 1,1 a $3 \mathrm{~cm}$ & 4,4 a $7,3 \mathrm{~cm}$ \\
\hline CEM II/AV & 0,9 a $2,3 \mathrm{~cm}$ & 3,5 a $5,4 \mathrm{~cm}$ \\
\hline CEM III & 0,4 a $1,2 \mathrm{~cm}$ & 1,7 a $2,8 \mathrm{~cm}$ \\
\hline \multicolumn{3}{|c|}{55 años } \\
\hline & Ambiente IIIa- 500m & Ambiente IIIc \\
\hline CEM I & $1,1 \mathrm{a} 3,1 \mathrm{~cm}$ & 4,5 a $7,5 \mathrm{~cm}$ \\
\hline CEM II/AV & 0,9 a $2,3 \mathrm{~cm}$ & 3,6 a $5,6 \mathrm{~cm}$ \\
\hline CEM III & 0,4 a $1,2 \mathrm{~cm}$ & 1,8 a $2,9 \mathrm{~cm}$ \\
\hline
\end{tabular}

Como se puede observar las diferencias en los recubrimientos de 50 a 55 años son mínimas y no pueden representar la seguridad frente a las incertidumbres que presenta este proceso.

\subsection{Verificación probabilista}

Desde un punto de vista práctico, se han efectuado análisis numéricos probabilistas sobre valores obtenidos por los procedimientos deterministas anteriormente expuestos, para poder verificar los extremos anteriormente indicados (6) (13) (17) (18).

Se elige para cada tipo de ambiente (IIIa-500 y IIIc), un hormigón de relación a/c $=0,4$, Contenido de cemento $350 \mathrm{~kg} /$ $\mathrm{m}^{3}$, tiempo 50 años y para cada tipo de cemento (CEM I, CEM II/A-V y CEM III), se recogen los recubrimientos deterministas y semiprobabilistas. 
En tales condiciones se efectúan los cálculos probabilistas, primero para conocer, cual es la variabilidad de los supuestos y para obtener una probabilidad de fallo de aproximadamente entre $11 \%$ y $13 \%$, con el recubrimiento semiprobabilis- ta. Posteriormente y aceptando una variabilidad mínima de $10 \%$ y probabilidades de fallo también en el orden anterior, estimar los recubrimientos necesarios, relacionándolos con los obtenido en el planteamiento determinista.

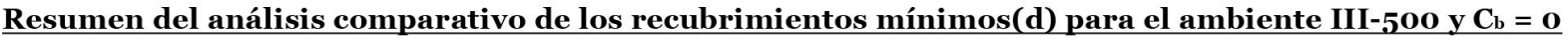

Cemento tipo CEM I.

AC $0.40-0.45-0.50-0.55$

$C C=-\left(\begin{array}{l}300 \\ 325 \\ 350 \\ 375 \\ 400\end{array}\right) \quad \mathrm{d}=-\left(\begin{array}{llll}20.49 & 21.72 & 27.30 & 30.48 \\ 18.01 & 19.10 & 23.99 & 26.80 \\ 15.62 & 16.56 & 20.82 & 23.25 \\ 13.32 & 14.12 & 17.75 & 19.82 \\ 11.10 & 11.76 & 14.78 & 16.51\end{array}\right)$

Cemento tipo CEM II/A-V

AVC $0.40-0.45-0.50-0.55$

$C C=\left(\begin{array}{l}300 \\ 325 \\ 350 \\ 375 \\ 400\end{array}\right) d:-\left(\begin{array}{llll}16.25 & 18.04 & 20.60 & 22.67 \\ 14.29 & 15.86 & 18.11 & 19.93 \\ 12.39 & 13.76 & 15.71 & 17.29 \\ 10.57 & 11.73 & 13.39 & 14.74 \\ 8.80 & 9.77 & 11.16 & 12.28\end{array}\right)$

Cemento tipo CEM III

AV $0.40-0.45-0.50-0.55$

$\alpha=-\left(\begin{array}{l}300 \\ 325 \\ 350 \\ 375 \\ 400\end{array}\right) \mathrm{d}=-\left(\begin{array}{llll}8.13 & 9.47 & 11.49 & 11.90 \\ 7.14 & 8.32 & 10.10 & 10.46 \\ 6.20 & 7.22 & 8.76 & 9.07 \\ 5.28 & 6.16 & 7.47 & 7.74 \\ 4.40 & 5.13 & 6.22 & 6.44\end{array}\right)$
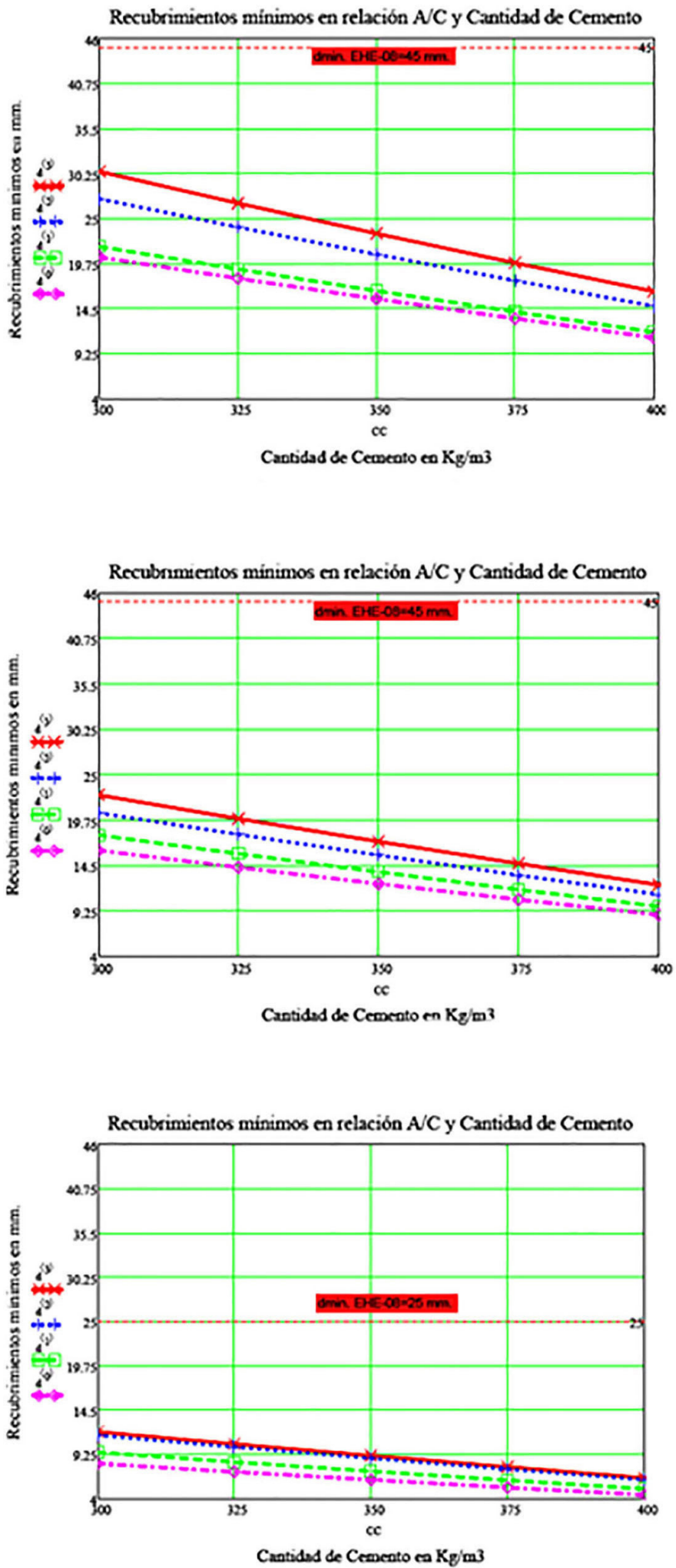

Figura 4. Estado comparativo de los recubrimientos para el ambiente IIIa-50o en función del tipo de cemento. 


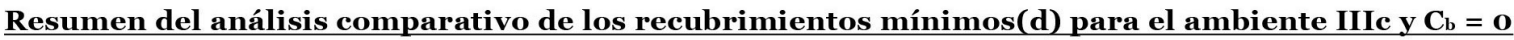

Cemento tipo CEM I

$C=\left(\begin{array}{l}300 \\ 325 \\ 350 \\ 375 \\ 400\end{array}\right) \quad \mathrm{d}=\left(\begin{array}{llll}49.07 & 52.02 & 65.39 & 73.01 \\ 47.76 & 50.63 & 63.64 & 71.06 \\ 46.50 & 49.29 & 61.96 & 69.18 \\ 45.28 & 48.00 & 60.33 & 67.37 \\ 44.10 & 46.75 & 58.77 & 65.62\end{array}\right)$

Cemento tipo CEM II/A-V

ACC $0.40-0.45-0.50-0.55$

$\alpha=\left(\begin{array}{l}300 \\ 325 \\ 350 \\ 375 \\ 400\end{array}\right) d=\left(\begin{array}{llll}38.93 & 43.21 & 49.35 & 54.31 \\ 37.89 & 42.06 & 48.03 & 52.86 \\ 36.89 & 40.94 & 46.76 & 51.46 \\ 35.92 & 39.87 & 45.54 & 50.11 \\ 34.99 & 38.83 & 44.35 & 48.81\end{array}\right)$

Cemento tipo CEM III

AC $0.40-0.45-0.50-0.55$

cC $=-\left(\begin{array}{l}300 \\ 325 \\ 350 \\ 375 \\ 400\end{array}\right)$ d $--\left(\begin{array}{llll}19.46 & 22.64 & 27.53 & 28.49 \\ 18.94 & 22.07 & 26.79 & 27.73 \\ 18.44 & 21.48 & 26.08 & 27.00 \\ 17.96 & 20.92 & 25.40 & 26.29 \\ 17.49 & 20.38 & 24.74 & 25.61\end{array}\right)$

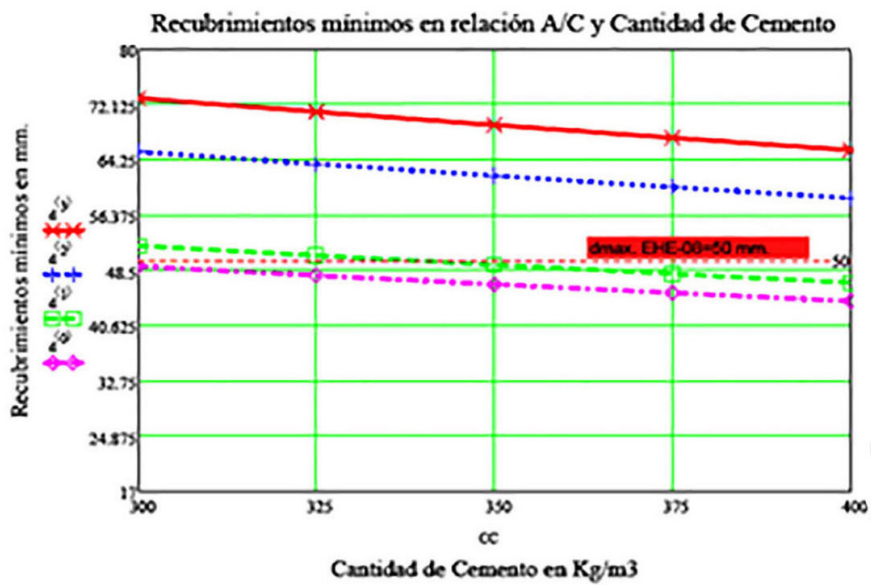

Recubrimientos minimos en relación A/C y Cantidad de Cemento
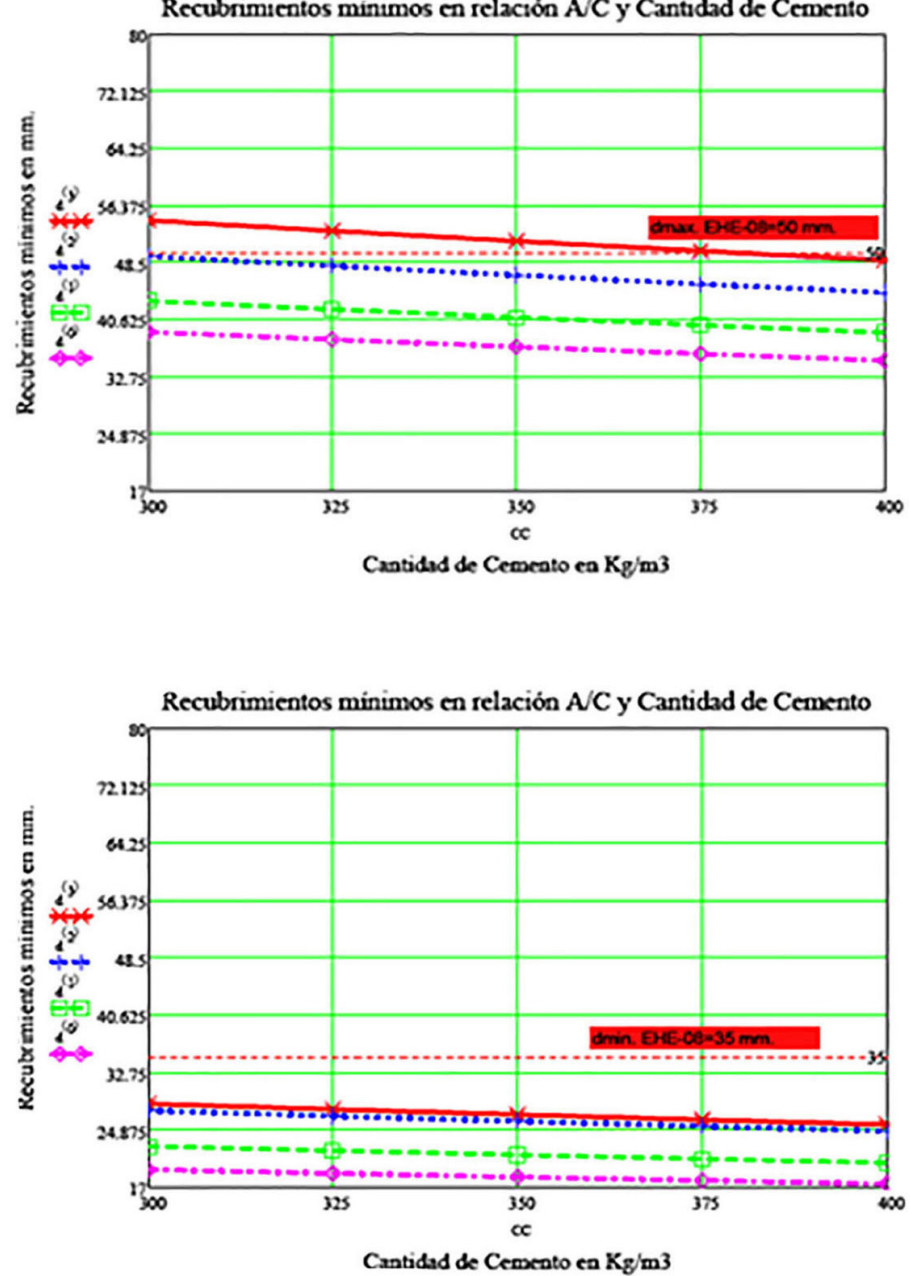

Figura 5. Estado comparativo de los recubrimientos para el ambiente IIIc en función del tipo de cemento.

Las conclusiones resultantes de tales cálculos son las siguientes:

1. Para los valores de los recubrimientos obtenidos con arreglo al anejo 9 de la EHE-08 y para 55 años, aceptando una probabilidad de fallo del orden del $12 \%$, (aproximadamente $\beta=1,1$ ), la variabilidad de las variables básicas queda reducida, aproximadamente a un $1 \%$, en el caso de ambiente IIIc y aproximadamente a 0,6 \% en ambiente IIIa-500.
2. Para conseguir probabilidades de fallo en el entorno del $10 \%$, con coeficiente de variación (CV) de todas las variables, del $10 \%$, los recubrimientos obtenidos en el modelo determinista a 50 años deberían multiplicarse por 1,5 para ambiente IIIa-500 y por 1,3 en ambiente IIIc.

En la Figura 6, se representa un ejemplo de los análisis efectuados, donde se observa que: 


\section{Ambiente IIla-CEM I-Cc=350 kg/m3- a/c=0.4-t=50 años}

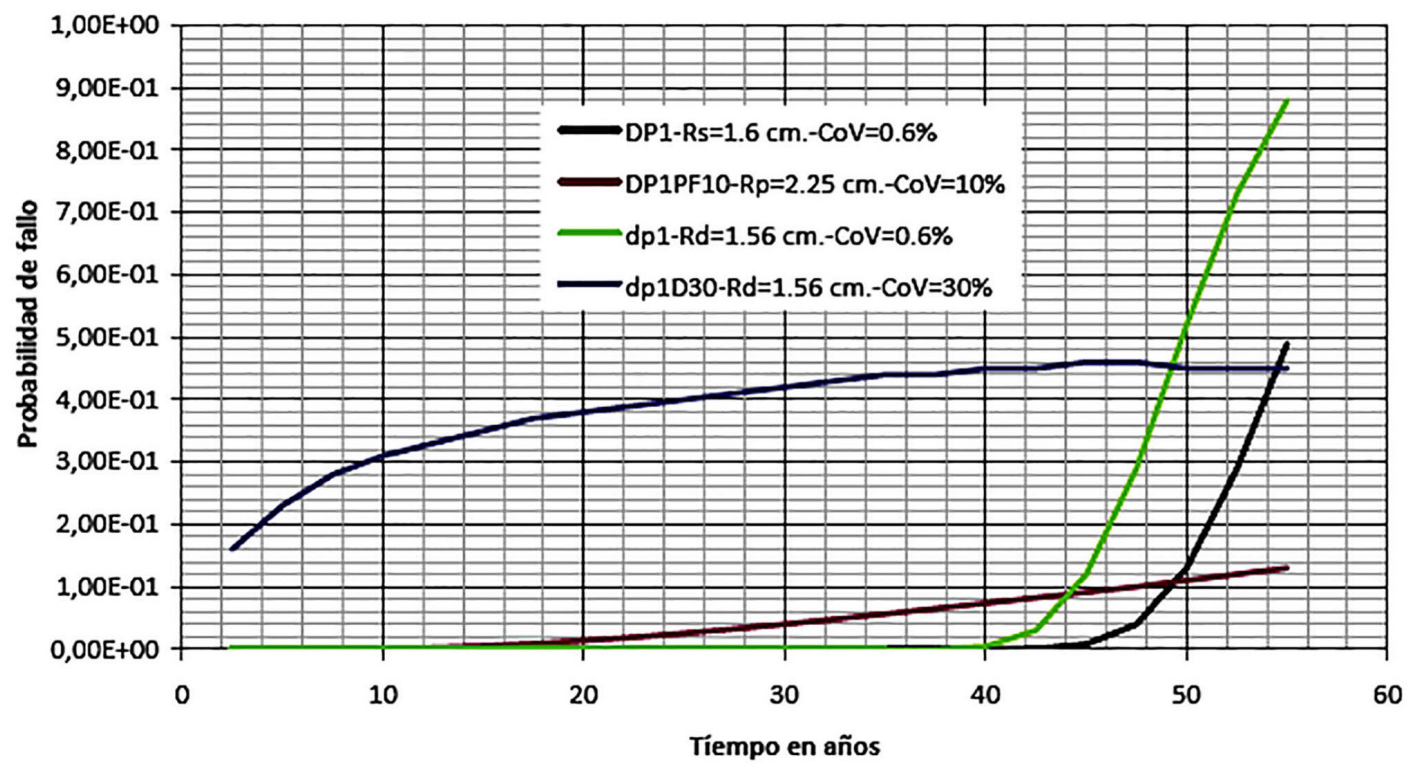

Figura 6. Probabilidades de fallo para recubrimientos semiprobabilisticos.

- Para un recubrimiento semiprobabilista $(\mathrm{Rs}=1,6 \mathrm{~cm}$, a 55 años), para obtener una probabilidad de fallo del orden del $10 \%$ a 50 años, el CV de las variables básicas debe ser del orden de 0,6 \% (línea negra). Se observa que la EHE - 08 exige conocer las variables básicas sin apenas grado de incertidumbre y que no va a existir ninguna probabilidad de fallo hasta aproximadamente los 45 años, cuando ésta empecerá a crecer exponencialmente (irreal).

- Comparando este recubrimiento con un valor determinista $(\mathrm{Rd}=1,56 \mathrm{~cm}$., a 50 años, línea verde); que es lo que hace la EHE-08 para dotar de seguridad al proceso; se ve que para este caso la probabilidad de fallo se sitúa en el 50 \% para la misma variabilidad (CV) de o,6 \%. En este caso, si se le da una variabilidad (CV) mayor, del $30 \%$, por ejemplo, a las variables (línea azul) se ve que a 50 años, se está aproximadamente en la misma probabilidad de fallo del $50 \%$, pero ahora la probabilidad de fallo crece desde el primer año.

- Si se quiere mantener una probabilidad de fallo del $10 \%$ a 50 años y una variación (CV) de las variables del $10 \%$ (por lo menos) el recubrimiento esperado probabilista sería de $\mathrm{Rp}=2,25 \mathrm{~cm}$. (línea granate). En esta la probabilidad de fallo va aumentando de manera progresiva hasta alcanzar el valor del $10 \%$ de una forma más real.

Por lo que comparando este valor con el determinista anterior: $\mathrm{Rp} / \mathrm{Rd}=1,44$.

De forma análoga se ha analizado, dentro de cada ambiente y para cada tipo de cemento, estas razones, presentando el siguiente resultado:

$\begin{array}{lll}\text { Ambiente IIIa-500. } & \text { CEM I, } & \text { Coef. }=1,44 \\ \text { Ambiente IIIa-50o. } & \text { CEM II/A-V, } & \text { Coef. }=1,53 \\ \text { Ambiente IIIa-500. } & \text { CEM III, } & \text { Coef. }=1,50 \\ \text { Ambiente IIIc } & \text { CEM I, } & \text { Coef. }=1,30 \\ \text { Ambiente IIIc } & \text { CEM II/A-V, } & \text { Coef. }=1,33 \\ \text { Ambiente IIIc } & \text { CEM III, } & \text { Coef. }=1,30\end{array}$

\section{APROXIMACIÓN PROBABILISTA DE LA DURABILIDAD}

La vida útil, $t_{\mathrm{L}}$, evaluada a través de un modelo de despasivación de las armaduras, ha de ser superior a la vida útil pretendida, $t_{\mathrm{g}}$. Es decir: $t_{\mathrm{L}}-t_{\mathrm{g}}>0$.

En estas condiciones se trata de obtener un coeficiente de seguridad de vida útil, $\gamma$, de tal forma que permita hacer esta estimación por un procedimiento determinista o semiprobabilista (19) (20) (21).

La probabilidad de que la vida útil estimada sea menor que un determinado valor, $t_{\mathrm{g}}$, aceptando una distribución de probabilidades log-normal será [1]:

$$
P\left(\operatorname{Ln}\left(t_{d}\right)<\operatorname{Ln}\left(t_{g}\right)\right)=\Phi(-\beta)
$$

es decir:

$$
F_{X}(x, \mu, \sigma)=\frac{1}{2} \operatorname{erfc}\left[\frac{-\operatorname{Ln}(x)-\mu}{\sigma \sqrt{2}}\right]=\Phi\left(\frac{\operatorname{Ln}(x)-\mu}{\gamma}\right)
$$

Por lo que el índice de fiabilidad será [2]:

$$
\beta=\frac{\mu(y)-\operatorname{Ln}\left(t_{g}\right)}{\sigma(y)}
$$

$t_{\mathrm{d}}$ : estimación de la vida útil de cálculo

$t_{\mathrm{g}}$ : vida útil de proyecto $\quad \gamma=\frac{t_{d}}{t_{g}}$

Para este tipo de distribución: 


$$
\begin{aligned}
& \mu(y)=\operatorname{Ln}\left(\mu\left(t_{d}\right)\right)-\frac{1}{2} \sigma^{2}(y) \\
& \sigma^{2}(y)=\operatorname{Ln}\left(1+\left[\frac{\sigma\left(t_{d}\right)}{\mu\left(t_{d}\right)}\right]^{2}\right) \quad \operatorname{Cov}=\frac{\sigma\left(t_{d}\right)}{\mu\left(t_{d}\right)}
\end{aligned}
$$

Por lo que sustituyendo [3] en [2] y operando:

$$
\begin{aligned}
& \beta \cdot \sigma(y)=\mu(y)-\operatorname{Ln}\left(t_{g}\right) \rightarrow \mu(y)=\beta \cdot \sigma(y)+\operatorname{Ln}\left(t_{g}\right) \\
& \operatorname{Ln}\left(\mu\left(t_{d}\right)\right)-\frac{1}{2}\left[\operatorname{Ln}\left(1+[\operatorname{Cov}]^{2}\right)\right]=\beta \cdot\left[\operatorname{Ln}\left(1+[\operatorname{Cov}]^{2}\right)\right]^{1 / 2}+\operatorname{Ln}\left(t_{g}\right) \\
& \operatorname{Ln}\left(\mu\left(t_{d}\right)\right)-\operatorname{Ln}\left(t_{g}\right)=\beta \cdot\left[\operatorname{Ln}\left(1+[\operatorname{Cov}]^{2}\right)\right]^{1 / 2}+\frac{1}{2}\left[\operatorname{Ln}\left(1+[\operatorname{Cov}]^{2}\right)\right] \\
& \operatorname{Ln}\left(\frac{\mu\left(t_{d}\right)}{t_{g}}\right)=\beta \cdot\left[\operatorname{Ln}\left(1+[\operatorname{Cov}]^{2}\right)^{1 / 2}+\frac{1}{2}\left[\operatorname{Ln}\left(1+[\operatorname{Cov}]^{2}\right)\right]\right.
\end{aligned}
$$

Como $\mu\left(t_{d}\right)=\gamma t_{g} \rightarrow \gamma=\frac{\mu\left(t_{d}\right)}{t_{g}}$

Tomando antilogaritmos:

$$
\gamma=e^{\left[\beta\left[\operatorname{Ln}\left(1+[\operatorname{Cov}]^{2}\right)\right]^{1 / 2}+\frac{1}{2}\left[\operatorname{Ln}\left(1+[\operatorname{Cov}]^{2}\right)\right]\right]}
$$

Como valores referenciales, la especificación LNEC-E 465 (22) (23) (24), establece:

$$
\begin{gathered}
\beta=1,5 \\
\operatorname{Cov}=0,5
\end{gathered}
$$

En estas condiciones la estimación del coeficiente de seguridad [4] para la ponderación de la vida útil se puede ver en las Figuras 7 y 8, donde se observa como varía respecto del índice de fiabilidad y coeficiente de variación. Para los valores referenciales, (22) (23) (24) anteriormente indicados en LNEC-E 465, el coeficiente se seguridad sería aproximadamente 2,3. Además se nota escasa dependencia, en términos relativos, del índice de fiabilidad, así para un índice de fiabilidad de 1,3 y coeficiente de variación o,5 el coeficiente de seguridad es 2,1.

Para conseguir un coeficiente de seguridad de 1,1 (el que establece la EHE-o8), habría que aceptar un coeficiente de variación menor de o,1. Valor claramente bajo que se corresponde con los análisis numéricos, sobre supuestos concretos, anteriormente indicados (apartado 2 de este artículo), tanto de forma probabilista como determinista.

\section{UTILIZACIÓN DE TÉCNICAS OPERACIONALES DE FIABILIDAD PARA LA ESTIMACIÓN DE COEFICIENTES PARCIALES}

Desde otro punto de vista, utilizando los factores de sensibilidad según la metodología FORM, tomada del anejo $\mathrm{E}$ de ISO 2394 (25) y considerando el tiempo de despasivación como una variable aleatoria se tendrían los siguientes valores:

- Valor característico $\left(t_{\mathrm{g}}\right)$ que se determina en forma de un fractil y un determinado coeficiente de variación dentro de una función de distribución de probabilidades [5], [8].

- Valor de cálculo $\left(t_{\mathrm{d}}\right)$ que constituye el punto de diseño o máxima verosimilitud y se determina en función del índice de fiabilidad $\beta$, sensibilidad de la variable a y coeficiente de variación [6], [9].

Pues bien, si se considerase la variable como lognormal, y un fractil que caracteriza la variable del $10 \%$, el valor característico será:

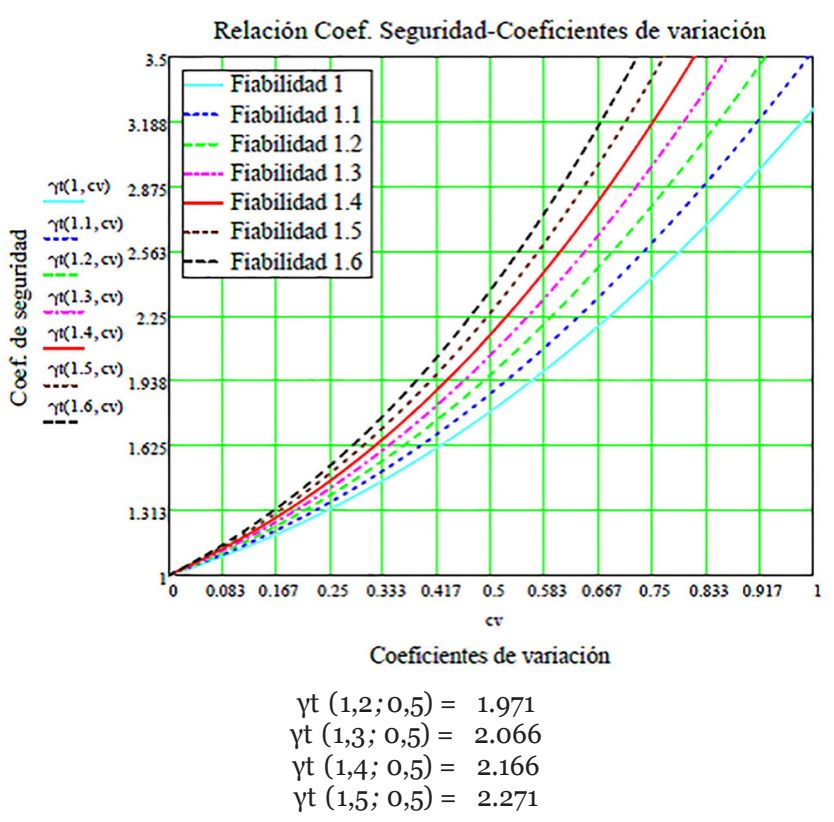

Figura 8. Relación entre el coeficiente de variación y el de seguridad para distintos índices de fiabilidad. 


$$
t_{g}=\mu(t) \cdot e^{\left[\frac{k\left[\operatorname{Ln}\left(1+C o v^{2}\right)\right]^{1 / 2}}{\left(1+C o v^{2}\right)^{1 / 2}}\right]}
$$

con $k=-1.282$ para un fractil del $10 \%$

El valor de cálculo será:

$$
t_{d}=\mu(t) \cdot e^{\left[\frac{-\beta \cdot \propto\left[\operatorname{Ln}\left(1+C o v^{2}\right)\right]^{1 / 2}}{\left(1+C o v^{2}\right)^{1 / 2}}\right]}
$$

Por tanto el coeficiente de seguridad será:

$$
\gamma=\frac{t_{d}}{t_{g}}
$$

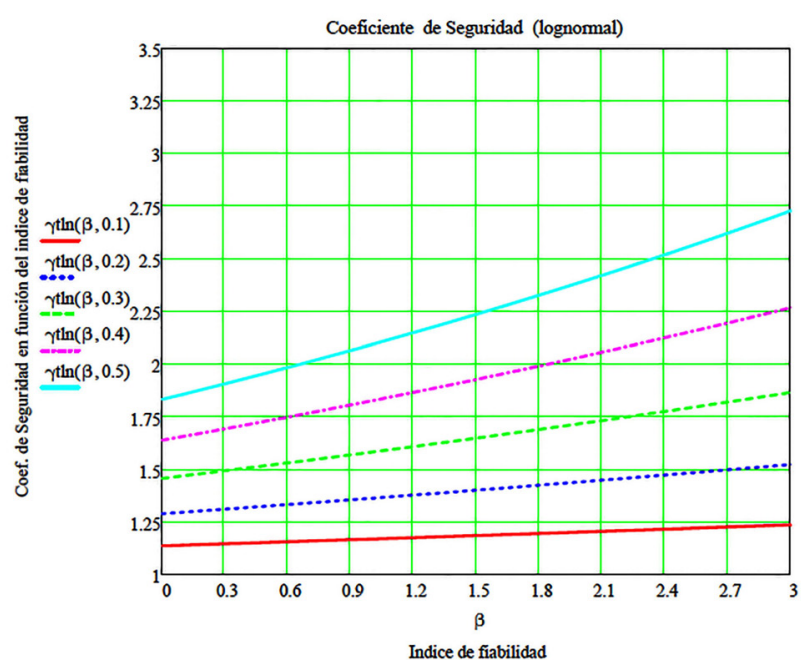

$\gamma \operatorname{thn}(1,5 ; 0,1)=1.185$

$\gamma \operatorname{tn}(1,5 ; 0,2)=1.401$

$\gamma \operatorname{tln}(1,5 ; 0,3)=1.648$

$\gamma \operatorname{thn}(1,5 ; 0,4)=1.926$

ytln $(1,5 ; 0,5)=2.234$

Figura 9. Relación entre el índice de fiabilidad y el coeficiente de seguridad para distintos coef. de variación.

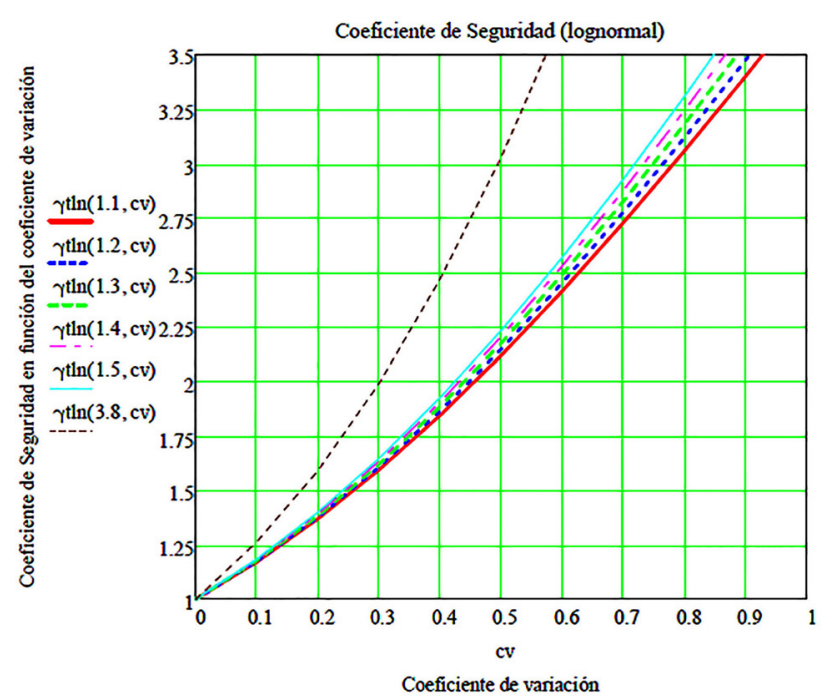

Figura 10. Relación entre el coeficiente de seguridad y el de variación para distintas fiabilidades.
Aceptando como factor de sensibilidad $\alpha=-0,28$ (según tabla E.3 Estandarización de $\alpha$-valores, de ISO 2394) (25).

Bajo estas condiciones se presentan los resultados en las Figuras 9 y 10.

Se observa prácticamente, la coincidencia de valores para el coeficiente de seguridad [7] en función del índice de fiabilidad y el coeficiente de variación.

Se hace más patente, que en el apartado anterior, la menor dependencia entre el índice de fiabilidad y el coeficiente de seguridad (para valores razonables de $\beta$, hay que pensar que se estaría ante un estado de servicio, $\beta \approx 1,1$ a 1,5 ).

Por último, si se considera ahora una distribución de probabilidades de la variable como tipo Gumbel, [7] con las mismas condiciones anteriormente indicadas.

El valor característico será:

$$
t_{g}=\mu(t)[1-\operatorname{Cov}(0,45+0,78 \operatorname{Ln}(-\operatorname{Ln}(0,1)))]
$$

Y el valor de cálculo será:

$$
t_{d}=\mu(t)[1-\operatorname{Cov}(0,45+0,78 \operatorname{Ln}(-\operatorname{Ln}(\Phi(-\beta \cdot \alpha))))]
$$

En estas condiciones el nuevo coeficiente de seguridad será:

$$
\gamma=\frac{t_{d}}{t_{g}}
$$

Estos cálculos se visualizan en las Figuras 11 y 12.

Los resultados corroboran los anteriormente indicados, ahora el coeficiente se seguridad [10] para un índice de fiabilidad

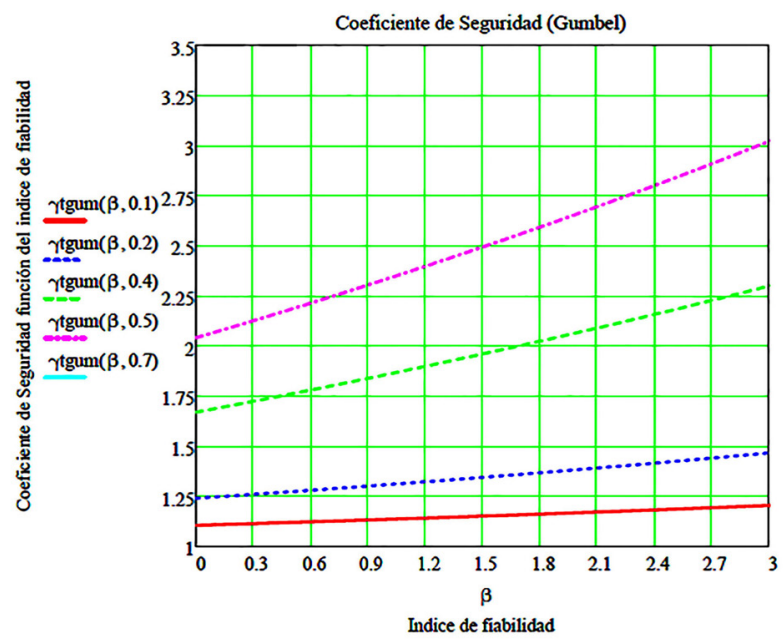

ytgum $(1,5 ; 0,1)=1.151$ $\gamma \operatorname{tgum}(1,5 ; 0,2)=1.345$ үtgum $(1,5 ; 0,3)=1.602$ үtgum $(1,5 ; 0,4)=1.96$ ytgum $(1,5 ; 0,5)=2.494$

ytgum $(1,3 ; 0,1)=1.144$ ytgum $(1,3 ; 0,2)=1.33$ $\gamma \operatorname{tgum}(1,3 ; 0,3)=1.576$ $\gamma \operatorname{tgum}(1,3 ; 0,4)=1.919$ ytgum $(1,3 ; 0,5)=2.429$
Figura 11. Relación entre el coeficiente de seguridad y el índice de fiabilidad para distintas variabilidades. 


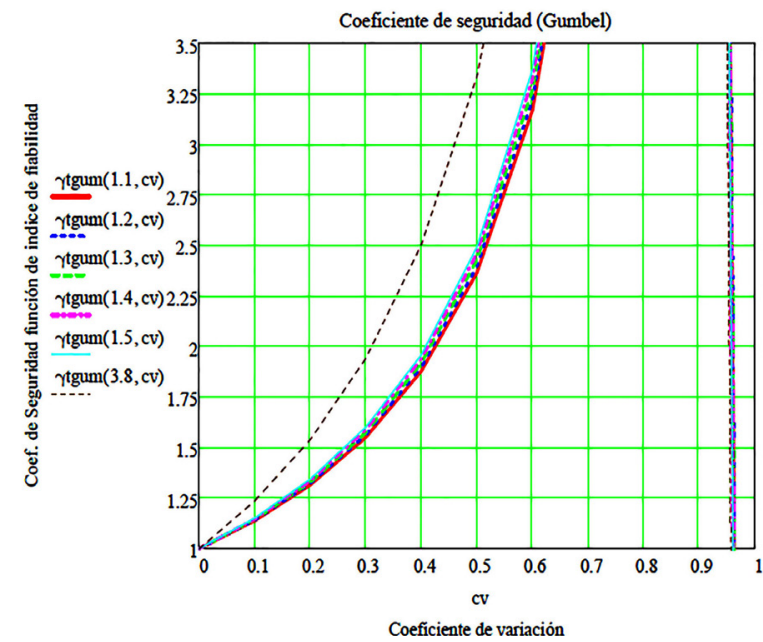

Figura 12. Relación entre el coeficiente de seguridad y el de variación para distintos índices de fiabilidad. de 1,5 y un coeficiente de variación de 0,5 es de, aproximadamente, 2,5 (con los planteamientos anteriores 2,3 ) y además con este tipo de distribución, aun se hace menor la dependencia del índice de fiabilidad y altamente dependiente del coeficiente de variación.

\section{CONCLUSIÓN FINAL}

La conclusión final se hace evidente. El valor de coeficiente de seguridad $(1,1)$, propuesto por la norma EHE-08, para ponderar la vida útil nominal para estimar la durabilidad de un elemento estructural sometido a la acción de cloruros, es claramente escaso y por tanto, no puede ponderar las incertidumbres que presenta el proceso.

En este artículo se propone un coeficiente de seguridad entre 2 y 2,5 sobre todo si como parece, los valores propuestos para las variables básicas en el anejo $9^{\circ}$, son valores medios.

\section{REFERENCIAS}

(1) Jean-Pierre, J. (Ed.). (2008). Eurocode 2 Commentary. Brussels: European Concrete Platform ASBL. http://www.efca. info/downloads/commentary_to_eurocode.pdf

(2) Rostam, S. (2006). Vida útil de las estructuras de hormigón. Cuadernos Intemac, (5): 13-21.

(3) ACI Committee 365. (2000). ACI 365.1.r-oo, Service Life Prediction- State of the Art Report. ACI.

(4) Walraven, J. (2010). Model Code 2010 - First complete draft. fib Bulletin, 1(55): 48-74.

(5) Matthews, S. L. (2010). Structural Concrete Textbook. fib Bulletin 3(53): 52-67.

(6) Faber, M. H. (2009). Curso "Risk and Safety in Engineering”. Zurich: Swiss Federal Institute of Technology - ETH Zurich. http://www.ibk.ethz.ch/emeritus/fa/education/ws_safety.

(7) Ferreira, R. M. (2004). Probability-Based Durability Analisis of Concrete Structures in Marine Environment (Tesis doctoral). Guimarães: Universidade do Minho - Escola de engenharia.

(8) Mendoza-Rangel, J. M., Castro-Borge, P. (2009). Validez de los conceptos y modelos vigentes de vida de servicio de estructuras de hormigón ante los efectos del cambio climático global. Situación actual. Materiales de construcción, 59(296): 117-124, doi: http://dx.doi.org/10.3989/mc.2009.46608.

(9) Mosquera-Rey, E. (2011). Probabilismo explícito en la corrosión de armaduras en las estructuras de hormigón sometidas al ambiente marino de la costa gallega (Tesis doctoral no publicada). A Coruña: Universidade da Coruña.

(10) Izquierdo, D. (2003). Bases de diseño para un tratamiento probabilista de los procesos de corrosión de armaduras en el hormigón (Tesis doctoral no publicada). Madrid: Universidad Politécnica de Madrid.

(11) Rostam, S. (1992). Tecnología moderna de durabilidad. Cuadernos Intemac, (5): 15, 29-37

(12) Tuutti, K. (1982). Corrosion of Steel in Concrete. CBI report 4: 82.

(13) Schiessl, P. (2006). Model Code for Service Life Design. fib Bulletin (34): 36-73.

(14) Tanner, P., Lara, C., Hingorani, R. (2007). Seguridad estructural. Una lucha con incertidumbres. Hormigón y acero, (245): 59-78

(15) López-Agüí, J. C. (2010). Determinación del recubrimiento mínimo de las armaduras en el hormigón estructural. Instituto Español del Cemento y sus Aplicaciones (IECA). http://www.concretonline.com/pdf/oohormigon/art_tec/ ieca_cemex_determinacin_recubrimiento_mnimo.pdf.

(16) Parametric Technology Corporation. Mathcad 14. http://es.ptc.com/product/Mathcad.

(17) Reliability Consulting Programs (RCP Munich). COMREL v8.1-TV-TI. http://www.strurel.de/index.html.

(18) Faber, M. (2001). Probabilistic Model Code. http://www.jcss.byg.dtu.dk/publications/probabilistic_model_code.aspx. JCSS.

(19) Gulvanessian, H., Holický, M. (1990). Annex C: Calibration Procedure. En Implementation of Eurocodes. Handbook 2: Reliability Backgrounds. http://www.eurocodes.fi/1990/paasivu1990/sahkoinen1990/handbook2\%5B1\%5D.pdf.

(20) Holický, M., Vrouwenvelder, T., Arteaga, A. (1990). Annex B: Methods of Structural Reliability. En Implementation of Eurocodes. Handbook 2: Reliability Backgrounds. http://www.eurocodes.fi/199o/paasivu1990/sahkoinen1990/ handbook2\%5B1\%5D.pdf.

(21) Holický, M. (1990). Annex A: Basic Statistical Concepts and Technique. En Implementation of Eurocodes. Handbook 2: Reliability Backgrounds. http://www.eurocodes.fi/1990/paasivu1990/sahkoinen1990/handbook2\%5B1\%5D.pdf.

(22) LNEC. (2007). E 464-2007. Betões. Metodologia prescritiva para uma vida útil de projecto de 50 e de 10o anos. Lisboa: Laboratório Nacional de Engenharia Civil.

(23) LNEC. (2007). E 465-2007. Betões. Metodologia para estimar as propriedades de desempenho do betão que permitem satisfazer a vida útil do projecto de estruturas de betão armado ou pré-esforçado sob as acções ambientais XC e XS. Lisboa: Laboratório Nacional de Engenharia Civil. 
(24) LNEC. (2004). E 463-2004. Determinação do coeficiente de difusão dos cloretos por ensaio de migração em regime não estacionário. Lisboa: Laboratório Nacional de Engenharia Civil.

(25) ISO. (1998). ISO 2394: 1998. General principles on reliability for structures. International Organization for Standardization. 